\title{
Protocol for Prevention and Control of COVID-19 (Edition 6)
}

\author{
National Health Commission of the People's Republic of China
}

To prevent and control COVID-19, the illness caused by the novel coronavirus 2019-nCoV, ensure "early detection, early reporting, early isolation, and early treatment," prevent further spread of the outbreak, reduce the rate of infection, improve treatment and cure rates, reduce the case fatality rate, protect people's safety and health, and maintain social stability, the Novel Coronavirus Pneumonia Prevention and Control Protocol, Edition 5, has now been revised and renamed the Protocol for Prevention and Control of COVID-19, Edition 6. This update is in accordance with the policy that COVID-19 is classified as a notifiable Category B infectious disease but regulated as a Category $\mathrm{A}$ infectious disease. The protocol update is based on the evolution of the epidemic across the country and research advances, and it will support the implementation of evidence-based practices with tailored and specific approaches for different regions of the country and stages in disease prevention and control.

\section{PURPOSE}

To prevent and control COVID-19 through provision of guidance of local efforts to detect and report COVID-19 cases and clusters in a timely manner, conduct epidemiological investigations and outbreak responses, and standardize management of close contacts.

\section{ETIOLOGY AND EPIDEMIOLOGICAL CHARACTERISTICS}

The novel coronavirus, 2019-nCov, is a $\beta$-type coronavirus, but its genetic characteristics are significantly different from SARS-CoV and MERS$\mathrm{CoV}$, the viruses that cause SARS and MERS, respectively. The 2019-nCoV is sensitive to ultraviolet rays and heat and can be killed by heating for 30 minutes at $56{ }^{\circ} \mathrm{C}$; lipid solvents such as ether, $75 \%$ ethanol, chlorine-containing disinfectants, peracetic acid, and chloroform can also inactivate the virus. Based on current epidemiological survey and research results, the incubation period is 1-14 days and generally in the range of 3-7 days. The main source of infection is COVID-19 patients, but asymptomatic individuals infected with 2019-nCoV may also infect others. The main modes of transmission are via droplets and direct contact. The possibility of aerosol transmission exists in relatively closed settings with exposure to high concentrations of aerosol for a long period of time. Other transmission routes need further investigation. Virtually the entire population is susceptible to 2019-nCoV infection.

\section{SURVEILLANCE CASE DEFINITIONS}

\section{Suspected Cases}

The definition considers both epidemiological history and clinical manifestations:

\section{Epidemiological history}

1. History of travel to or residence in Wuhan and its surrounding areas, travel to other communities in China where cases have been reported, or travel to other countries/regions with severe outbreaks outside China within 14 days prior to the onset of the disease;

2. Contact with an individual infected with 2019$\mathrm{nCoV}$ (who tested positive with a nucleic acid test) within 14 days prior to the onset of the disease;

3. Contact with patients with fever or respiratory symptoms from Wuhan, Wuhan's surrounding areas, communities where confirmed COVID-19 cases have been reported, or from other countries/regions with severe outbreaks outside China within 14 days before the onset of the disease; or

4. Clustered cases (two or more cases with fever and/or respiratory symptoms in a small area such as in families, offices, schools, workplaces, and other gatherings within 14 days).

\section{Clinical manifestations}

1. Fever and/or respiratory symptoms;

2. Radiographic imaging consistent with COVID19 pneumonia;

3. Normal or decreased WBC count, or normal or decreased lymphocyte count in the early stages of illness.

A suspected case is defined by having any of the epidemiological history criteria plus any two clinical manifestations or by having all three clinical 
manifestations if there is no clear epidemiological history.

\section{Confirmed Cases}

Suspected cases with one of the following etiological or serological pieces of evidence:

1. Real-time fluorescent RT-PCR positive result for 2019-nCoV nucleic acid;

2. Viral genetic sequence that is highly homologous to 2019-nCoV;

3. 2019-nCoV virus-specific $\operatorname{IgM}$ and $\operatorname{IgG}$ detectable in serum, 2019-nCoV virus-specific $\operatorname{IgG}$ is detectable, or there is at least a 4 -fold increase in $\operatorname{IgG}$ between paired acute and convalescent sera.

\section{Asymptomatic Infected Persons}

Asymptomatic individuals with etiological detection of $2019-\mathrm{nCoV}$ in respiratory specimens or specific IgM detected in serum. These individuals may be found through tracing of close contacts, investigation of clusters, and tracing of sources of infection.

\section{Clusters of Cases}

Clusters of cases refer to 2 or more confirmed COVID-19 cases or asymptomatic 2019-nCoVinfected persons in a small area such as in families, offices, schools, workplaces, and other gatherings within a 14-day period, in which there exists the possibility of human-to-human transmission or common exposure.

\section{Close Contacts}

People who had unprotected, close contact with a confirmed or suspected case within two days prior to illness onset or with an asymptomatic 2019-nCoVinfected person within two days before obtaining clinical samples from the asymptomatic infected person.

\section{PREVENTION AND CONTROL MEASURES}

\section{Precision Prevention and Control Tailored to Specific Areas and Levels}

In accordance with the Law of the People's Republic of China on the Prevention and Treatment of Infectious Diseases and the Regulations on Emergency Response to Public Health Emergencies, precise, tailored prevention and control measures are to be implemented for different regions and at different levels. Epidemic risk level shall be assessed for each county/district (as a unit) based on its demographic and epidemiological situation, and appropriate prevention and control strategies shall be determined.

In low-risk areas, the strategy is to "strictly prevent importation". This includes strengthening tracking and management of people coming in from areas with severe outbreaks and high-risk areas and enhancing health monitoring and services. Fever clinics should strengthen monitoring, detection, and reporting of outpatients with fever, and the CDCs should conduct timely epidemiological investigations and tracking and careful management of close contacts. The government should provide guidance to both urban and rural communities, government agencies, enterprises, and public institutions to strictly implement community prevention and control measures, improve environmental hygiene, and promote disease prevention knowledge and skills to the general public.

In medium-risk areas, the strategy is "to prevent importation and stop local transmission". This includes the measures for low-risk areas, augmented with preparation for medical treatment, personnel, materials, and venues required for disease prevention and control efforts, and isolated medical observation and management of close contacts. School classrooms, building units, factory workplaces, and workplace offices will serve as the smallest units of regulation. Resources, such as personnel for prevention and control and tailored measures, can be identified and located based on a case-by-case review, epidemiological investigation, and epidemic situational analysis. Townships, streets, and urban and rural communities without confirmed cases may implement prevention and control measures of low-risk areas.

In high-risk areas, the strategy is "to stop local transmission, prevent exportation, and implement strict prevention and control measures". In addition to measures for medium-risk areas, this strategy involves stopping activities involving gatherings of people and implementing regional traffic controls-with approval and in accordance with law and procedures. Every county should conduct comprehensive screenings of patients with fever; admit and manage in a timely manner suspected cases, confirmed cases, and asymptomatically-infected patients; and isolate and place close contacts under medical observation. Disinfection shall be conducted in sites with 
community transmission or clustered outbreaks in urban residential areas or rural villages, and control measures shall be taken to restrict the gathering, entry, and exit of people from the above sites.

It is essential to conduct dynamic research and analysis, adjust risk levels in a timely manner, and reduce emergency response levels or terminate emergency responses after the case numbers decline steadily and the risk of epidemic spread is effectively controlled.

\section{Early Detection}

Healthcare facilities at all levels should raise their awareness of diagnosing and reporting COVID-19 cases. For individuals with respiratory symptoms such as fever and dry cough and digestive tract symptoms such as diarrhea of unknown cause, their epidemiological history should be assessed, and expert, joint consultations arranged during the time of specimen collection and testing.

Primary level organizations or employers should put great effort in screening people who travelled to or resided in Wuhan and its surrounding areas within 14 days, people who travelled to or resided in communities where confirmed cases have been reported, and people who have respiratory symptoms, fever, chills, fatigue, diarrhea, conjunctival congestion, or other symptoms consistent with COVID-19 illness. These individuals are key risk groups for screening, and their clinical sampling and testing should be performed by professional institutions.

Existing surveillance networks for pneumonia of unknown etiology (PUE), influenza-like illness (ILI), and hospitalized severe, acute, respiratory illness (SARI) should be employed to strengthen etiological surveillance.

Port health quarantine should be strengthened by strictly implementing temperature monitoring and medical inspection at inbound and outbound ports. For people with respiratory symptoms such as fever and dry cough and digestive tract symptoms such as diarrhea, epidemiological investigation and medical screening should be conducted, and specimens collected and pathogenic testing performed accordingly.

The health status of close contacts should be monitored. Patients with respiratory symptoms such as fever and dry cough and digestive tract symptoms such as diarrhea should be transferred in a timely manner to the designated healthcare facilities with their specimens already collected and pathogenic testing completed.

\section{Early Reporting}

Case reporting. When suspected cases, confirmed cases, or asymptomatically-infected individuals are identified, healthcare facilities at all levels and of all types should report the cases immediately via the online direct reporting system. CDCs, upon receiving a report, shall conduct an investigation immediately, verify the report, and complete a three-level confirmation and review within two hours in the online direct reporting system. Healthcare facilities without online direct reporting capacity should make a prompt report to the local county/district CDC and send a notifiable disease report card within two hours. The local county/district CDC should make an online direct report upon receiving such a notification and ensure the accuracy of all information.

Updating reports. Once suspected cases are confirmed or ruled out, the information should be updated in a timely manner. If previously asymptomatic but infected persons develop clinical manifestations, they should reclassified as "confirmed cases" in a timely manner. "Clinical severity" of all cases should be updated with the progression of illness, with the most severe condition of the case as its final severity designation. For individuals who died of COVID-19, the date of death should be recorded within 24 hours.

When reporting asymptomatically-infected cases, the "collection date of a positive specimen" should be used as the "date of onset", and the "positive detection date" should be used as the "date of diagnosis". If an "asymptomatically-infected person" has been updated to a "confirmed case", the "date of onset" should be the date at which clinical symptoms appeared.

Reporting of emergency events. According to the requirements of the National Public Health Emergency Response Contingency Plan and the National Public Health Emergency Related Information Reporting and Management Rules (Trial Edition), the index COVID19 confirmed case or cluster in a county/district should be reported within two hours by the local CDC in the jurisdiction through the online direct reporting system for public health emergencies. The emergency level can be initially categorized as "unclassified" and should be updated in a timely manner based on investigation findings and assessments.

\section{Early Isolation}

Case management. Suspected and confirmed cases should be isolated and treated in designated healthcare 
facilities. Suspected cases should be isolated and treated in single rooms and can be ruled out as a suspected case following 2 consecutive negative virus nucleic acid tests with at least a 24-hour interval between samplings and negative IgM and IgG tests 7 days after onset of isolation.

Management after discharge. After a patient reaches discharge criteria and is discharged, the patients should undergo a 14-day isolation and have their health status continuously monitored throughout the 14 days. When possible, provincial-level administrative divisions are encouraged to strengthen follow-up observation of discharged patients and testing of their respiratory specimens; those testing positive should be put in centralized isolation for medical observation with their information submitted to China CDC.

Management of asymptomatic infected persons. Asymptomatic infected persons should be put under centralized isolation for 14 days. Those testing negative with two consecutive virus nucleic acid tests (with at least 24-hour interval between samplings) can be released from isolation.

\section{Early Treatment}

Healthcare facilities at all levels and of all types should transfer suspect cases to designated hospitals in a timely manner. Designated hospitals will be well prepared for case treatment in terms of personnel, medicines, facilities, equipment, and personal protective equipment and will provide treatment according to the most up-to-date COVID-19 diagnosis and treatment protocol. Efforts should be made to ensure that "all in need are tested, admitted, treated, and isolated" in order to improve admission and cure rates and to reduce infection and case fatality rates.

\section{Epidemiological Investigation}

Case investigation. The county/district CDC, upon receiving a report, should complete an epidemiological investigation of cases and asymptomatically-infected persons within 24 hours. The investigation should be conducted in accordance with requirements set out in the Guidelines for COVID-19 Epidemiological Investigation issued by China CDC. Tracing and registration of close contacts should be conducted following requirements in the Guidelines for COVID19 Close Contact Management, and for suspected cases, the patient's basic information and his or her close contacts should be registered.

Cluster investigation. The county/district CDC should conduct immediate investigation of suspected clusters identified in the online-reported information and case investigation findings, following requirements set out in the Guidelines for COVID-19 Epidemiological Investigation, as issued by China CDC.

Information reporting. The county/district CDC, upon completion of a case investigation of a confirmed case or asymptomatically-infected person or an investigation of a suspected cluster, should submit the case investigation form and investigation report to the online reporting system in a timely manner.

\section{Close Contact Tracing and Management}

Tracing and management of close contacts are organized and implemented by the county/district health authorities along with other relevant departments. Close contacts are to be put under centralized isolation and medical observation; if this is not feasible, home isolation and medical observation can be used instead. Close contacts should be monitored at least twice a day, recording their body temperature and recording whether they have respiratory symptoms such as fever and dry cough or digestive tract symptoms such as diarrhea. The medical observation period for close contacts is 14 days after the last unprotected contact with a case or with an asymptomatic infected person. If a suspected case is ruled out, his or her close contacts can be removed from medical observation in accordance with requirements set out in the Guidelines for COVID-19 Close Contact Management issued by China CDC.

\section{Specimen Collection and Lab Testing}

Healthcare facilities receiving COVID-19 cases should collect relevant clinical specimens in a timely manner. Laboratories testing specimens (including eligible healthcare facilities, CDCs, or third-party labs) should report test results within 12 hours. Specimen collection, transportation, storage, and testing should be conducted strictly in accordance with the requirements set out in the Technical Guidelines for COVID-19 Laboratory Testing issued by China CDC.

All original specimens from clusters of five or more COVID-19 cases should be sent to the China CDC for verification and confirmation.

\section{Strengthen Prevention and Control Measures Targeting Key Settings, Institutions, and Populations}

It is essential to strengthen multi-sectoral, joint 
prevention and control mechanisms to minimize public gathering activities and to implement measures such as ventilation, disinfection, and taking body temperature in places with large population flows such as train stations, airports, ports, shopping malls, public toilets, and closed vehicles such as cars, trains, and airplanes.

Health authorities should guide businesses to arrange for their employees to return to work in phases and in logical groupings; to strictly conduct ventilation, disinfection, body temperature detection, and other prevention and control activities; to provide employees with necessary personal protective equipment and adopt strategies of partitioning operations and non-grouped dining; to effectively reduce the concentrations of people; and to provide health education among migrant workers from rural areas and strengthen temperature screening before returning to work. Once an abnormal situation is detected, timely reporting, screening, and identification and response measures should be implemented to prevent people from leaving the area.

After schools and kindergartens reopen, health authorities shall provide health education, guide health management of returning teachers and students, diligently ventilate and disinfect the classrooms, and supervise implementation of prevention and control measures such as morning/afternoon checks, tracing and registration of illness-related absences, and attendance. When an epidemic report is received, epidemiological investigation, response measures, and guidance on disinfecting the affected area should be urgently conducted.

For special institutions such as nursing homes, welfare institutions for the disabled, and places of detention, the government should standardize management of personnel entry and exit, strict ventilation, daily cleaning and disinfection, and other health measures and strengthen personal protection measures, health monitoring, and daily management of incapacitated and semi-incapacitated individuals.

It is essential to implement health inspections and quarantine at ports for people entering China, including enhancement of health management of people coming to China from countries and regions with serious epidemic situations. Activities include screening, investigation, diagnosis, treatment, and medical observation of suspected cases, confirmed cases, and close contacts to strictly prevent cross-border spread of the epidemic.

\section{Nosocomial Infection Control, Disinfection of Specific Settings, and Personal Protection}

Healthcare facilities should follow the requirements set out in the technical protocol for COVID-19 control and prevention in healthcare facilities to strictly implement nosocomial infection control and prevention measures. This includes following the requirements in the Technical Guidelines for Disinfection in Healthcare Facilities and Hospital Air Purification Management Guidelines to clean and disinfect medical equipment, contaminated articles, surfaces, and floors, and to follow the Medical Waste Disposal Regulations and the Measures for Medical Waste Management in Healthcare Facilities for the disposal and management of medical wastes.

It is essential to implement effective disinfection of places used by COVID-19 patients and asymptomatic infected persons such as homes, isolation wards in healthcare facilities, transportation vehicles, and medical observation sites. It is essential to ensure effective protection of professionals who carry out epidemiological investigations, who work in isolation wards and medical observation sites, who transport cases, infected persons, or deceased persons, and who are involved in environmental cleaning and disinfection, specimen collection, or laboratory work. For relevant requirements, see Technical Guidelines for the Disinfection of Special Sites for COVID-19 and Guidelines for Personal Protection of Specific Groups from COVID-19 issued by China CDC.

\section{Public Education and Risk \\ Communication}

It is essential to disseminate knowledge about COVID-19 and its prevention and control. Health education and risk communication shall be enhanced for key populations to help guide the public on personal protection to reduce possible contact or exposure. With progress in epidemic prevention and control efforts and increasing understanding of COVID-19, health education strategies should be updated and corresponding scientific public education should be organized in a timely manner. Activities include public opinion monitoring, promptly responding to concerns and questions of the public, and conducting risk communication on epidemic prevention and control. 


\section{SUPPORTIVE MEASURES}

\section{Strengthening Organization and Leadership}

Local governments should strengthen their leadership in response to the outbreak, ensure availability of funding and materials, and implement prevention and control measures following the principles of "prevention first, integration of prevention and treatment, scientific guidance, and timely treatment".

\section{Enhancing Joint Prevention and Control Efforts}

Cross-sectoral information sharing and regular discussion and assessment of the epidemic trends should be implemented and strengthened. Health authorities at all levels are responsible for overall guidance of the epidemic control work. CDCs at all levels are responsible for case surveillance, epidemiological investigations, close contact management, and laboratory testing. Healthcare facilities at all levels are responsible for case finding and reporting; isolation, diagnosis, treatment, and clinical management; and specimen collection and nosocomial infection control and prevention.

\section{Strengthening Capacity Building}

Technical training of health professionals in medical and health facilities on COVID-19 with a focus on proactive prevention first shall be implemented. Strengthening scientific research and giving full play to the role of information technology in the prevention and control of infectious diseases is essential, as is conducting careful investigations on COVID-19 transmission dynamics, clinical features, and strategy assessment to provide scientific evidence to optimize prevention and control strategies. The use of traditional Chinese medicine in the prevention and treatment of infectious diseases is encouraged and supported.

Acknowlegement China CDC Weekly would like to thank Xijuan $\mathrm{Fu}$ of the WHO China Office and Lance Rodewald and Liping Wang of China CDC for their contributions in translating and editing this document (1).

doi: $10.46234 /$ ccdcw 2020.082

Submitted: March 08, 2020; Accepted: March 10, 2020

\section{REFERENCES}

\footnotetext{
1. National Health Commission of the People's Republic of China Protocol for prevention and control of COVID-19 (Edition 6). http://www.nhc.gov.cn/jkj/s3577/202003/4856d5b0458141fa9f376853 224d41d7.shtml. [2020-3-7]. (In Chinese).
} 